\title{
A Novel Centralized Network for Sensing Spectrum in Cognitive Radio
}

\author{
Hongjian Sun, D.I. Laurenson, J.S. Thompson \\ Institute for Digital Communication \\ Joint Research Institute for Signal \& Image Processing \\ School of Engineering and Electronics \\ University of Edinburgh, Edinburgh, UK \\ Email: \{H.Sun, Dave.Laurenson, John.Thompson\}@ed.ac.uk
}

\author{
Cheng-Xiang Wang \\ Joint Research Institute for Signal \& Image Processing \\ School of Engineering and Physical Sciences \\ Heriot-Watt University, Edinburgh, UK \\ Email: cheng-xiang.wang@hw.ac.uk
}

\begin{abstract}
Spectrum sensing plays a paramount role in cognitive radio (CR), which is widely agreed to be the most promising method for alleviating the symptom of RF spectral scarcity. In this paper, we first analyze the effects of multipath/shadowing on a CR system. Furthermore, we build models for the primary user system and the CR user system seperately. For the former model, we develop the Possible Conversation Zone to represent the $C R$ cooperative detectable zone. For the latter model, we construct a novel centralized network, called the Spider-Net Sensing Network (SNSN), to implement controllable sensing resolution and reliable sensing while avoiding harmful interference from other CR users. Finally, the numerical results show that our proposed SNSN outperforms one without a grid structure using the same combining scheme.
\end{abstract}

\section{INTRODUCTION}

$\mathrm{CR}$ is commonly recognised as one of the most promising methods to solve the scarcity of RF spectrum resource, which is evident in the National Telecommunication and Information Administration (NTIA) frequency allocation graph. When the concept was first introduced by Mitola [1], it was initially envisioned as renting sparse spectrum to secondary users by an opportunistic but polite scheme, namely, the secondary users should not impose harmful interference on the communication of the primary users. Tailored for efficiently exploiting spectrum resource and seeking comments on spectrum policies, the Spectrum Policy Task Force (SPTF) within the Federal Communications Commission (FCC) was formed in June 2002. In the same year, SPTF reported that under the existing fixed spectrum allocation policy, the temporal and geographic spectrum utilization efficiency ranges from $15 \%$ to $85 \%$ [2]. For the purpose of providing high bandwidth to wireless users via dynamic spectrum access, the NeXt Generation communication networks program, supported by the Defense Advanced Research Project Agency (DARPA), was developed to implement the spectrum policy based on CR Networks. Furthermore, the IEEE organized a new working group, known as the Wireless Regional Area Network (WRAN, IEEE 802.22), to develop standards, on one hand, for the usage of unlicensed spectrum, typically the Industrial, Scientific and Medical (ISM) bands, and on the other hand, for the sharing of licensed bands, currently utilized by UHF/VHF TV from $54 \mathrm{MHz}$ to $862 \mathrm{MHz}$. For the sake of the former bands, reference [3] presented a CR approach for the usage of Virtual Unlicensed Spectrum (CORVUS) to develop new strategies of sharing unoccupied bands with CR users. With regard to the presence of licensed user in the latter bands, Spectrum Pooling was proposed to gather available band information in a central base-station by a boosting protocol based on Orthogonal Frequency Division Multiplexing (OFDM) [2].

$\mathrm{CR}$ is viewed as an intelligent radio for its functions of spectrum sensing, spectrum analysis and spectrum decision. What is remarkable is that the CR not only detects the RF stimulus, but also senses the spectrum, intelligently, adaptively, reliably and efficiently as summarized in [4]. Generally, three kinds of detection methods are employed to sense the spectrum. Firstly, matched filter detection [5] is the optimal detector in the background of stationary Gaussian noise since it achieves the maximum Signal-to-Noise Ratio (SNR). Nevertheless, as it may not be easy to get prior knowledge of the primary user signal, we see that matched filter detection encounters the obstacle of insufficient prior knowledge for noncoherent detection. What is worse is that for good performance of matched filter detection, perfect synchronization should be guaranteed, which would consume additional time and hardware. Secondly, the energy detection method [6] is a suboptimal approach for any type of signals. However, one of the major advantages of energy detection is that the computational complexity is inversely proportional to $S N R^{2}$. In spite of that, the uncertainty of the power level for noise creates a new hurdle for robust detection, called the SNR wall, below which a detector can not perform reliable detection even with an infinite sampling time. Lastly, cyclostationary feature detection [7], [8] was proposed due to its outstanding performance even in the worst-case scenario of large power level uncertainty of noise. It uses the characteristic that noise has no correlation, whereas any man-made signal have some degree of correlation. However, the minimum number of samples required for detection is much more than that for energy detection, which means longer observation time and higher calculation complexity are required than those for the energy detection approach.

The most important aim of CR is to use spectral resource efficiently, by exploiting temporal or spatial spectrum accesses. Unfortunately, until now, joint consideration of the temporal 
and spatial utilization efficiency of the spectrum remains unknown. This is the focus for the remainder of this paper. The rest of the paper is organized as follows: In Section II, we introduce the primary system model and CR system model to set a specific cooperative detectable zone for CR users, and present a novel centralized network for sensing spectrum, called SNSN, which has the functions of controlling sensing resolution, reliable detection of the primary system and avoiding interference from other CR users. The combining method and experimental environments are described in Section III. Numerical results are presented in Section IV, and the paper's conclusions given in Section V.

\section{THE SNSN}

In a system where CR users and Primary users coexist, sensing the spectrum is a significant task for the CR system, because the primary users are licensed to have the right to use specific bandwidths. The problem is that, in addition to signal attenuation, CR users may suffer the effects of multipath/shadowing, which will make the practical received power lower. In other words, the CR system should have higher detection sensitivity than the primary system. For detection considerations, the $\mathrm{CR}$ sensitivity requirement should be equal to or lower than the expected practical received power. However, if the $\mathrm{CR}$ sensitivity requirement is under the noise floor, how can we detect the primary system? In the following subsections, we build a primary system model and a CR system model to combine useful sensing information for decreasing the detection sensitivity requirements of the CR system.

\section{A. Primary System Model}

In practice, we hope that primary users occupy a defined region for communication, inside of which the CR users should not cause detrimental interference to the primary system. In [9], the No-talk-Zone model was constructed when considering many factors including the possible receiver location, shadowing/fading and the accumulated interference from multiple CR users. For the sake of practical application, we upgrade the above model to our primary system model as shown in Fig.1. Based on the path-loss information of the transmitted power from the primary system, we set the location of primary transmitter as a circle centre and draw a Possible Conversation Zone, in which the primary system wireless connections probably exist. Meanwhile, near the primary receiver, we use a Notalk-Zone to avoid the interference from CR users, in which the $\mathrm{CR}$ users should be kept silence. But the primary receiver may be located anywhere in the possible conversation zone, thus, we set a protection guard band for ensuring no interference from the $\mathrm{CR}$ system. If the primary receiver is near the primary transmitter, the No-talk Zone will be very small. And if the primary receiver is far from the primary transmitter, the Notalk Zone will be larger. The Possible Conversation Zone is also the cooperative detection zone for CR users.

The majority of existing methods for spectrum sensing, e.g., energy detection or cyclostationary feature detection, are based

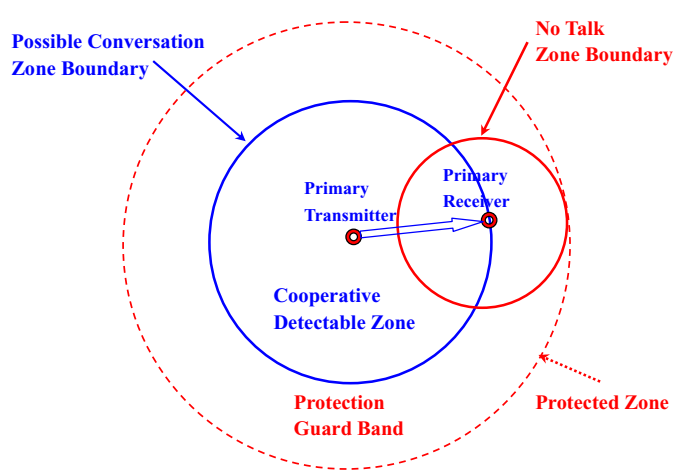

Fig. 1. The model of the primary system.
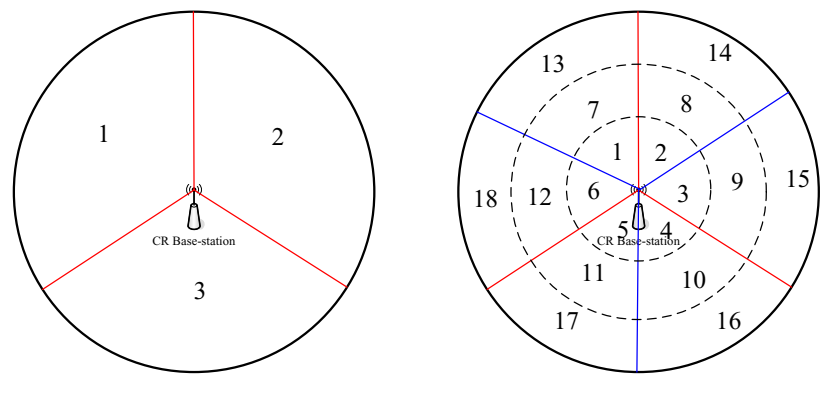

Fig. 2. The model of the CR system.

on detecting the primary transmitter in a specific band. In contrast, the primary user receiver detection method has some challenges in passive receiver systems, such as a Television Receiver. In other words, it is important to accurately detect the location and power level of the primary transmitter rather than the primary receiver. Accordingly, the possible conversation zone is more inclusive than the No-talk-Zone for our detection method, as we can keep enough space for the different level sensitivities of the primary receiver, particularly in the case of that we know the primary transmitter information.

\section{B. CR System Model}

Inspired by the principle that different sizes of grids in a spider-web can be used for preying on different kinds of insects, while other parts of the net can also be concurrently used to catch other flies, we attempt to weave a spider-web like network to sense the presence of the primary system, as shown in Fig. 2. It is a centralized network for detecting the Possible Conversation Zone.

At the centre of the net, the CR base-station has the function of constructing the SNSN. By using sectorised antennas, or Angle-of-Arrival (AOA) estimation algorithms, with the aid of multiple antennas, the $\mathrm{CR}$ base-station can distinguish the directions of the CR users. That means the the entire region could be divided into several pieces of pizza-like segments. Meanwhile, by employing Time-Difference-Of-Arrival (TDOA), the CR base-station can further divide these pieces into several circles as illustrated in Fig. 2. 


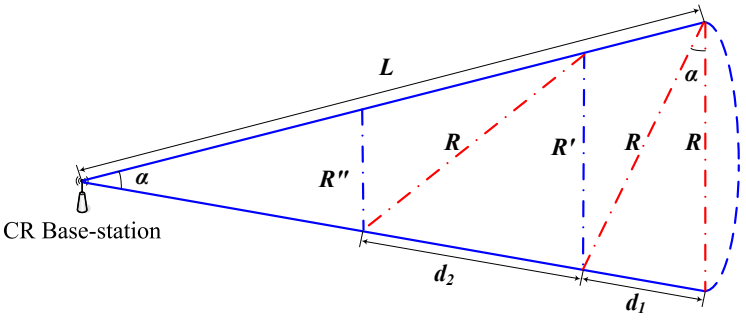

Fig. 3. Construction of the Spider-Net Sensing Network

\section{Procedure for Constructing the SNSN}

In order to capture the possible conversation zone, we try to connect the SNSN parameters with the range of possible conversation zones. We assume that we know some essential parameters of the primary system, for example the maximum communication distance of the primary transmitter, path-loss parameters and the sensitivity of primary receivers. If these requirements can be satisfied, the SNSN can achieve better performance for sensing spectrum. The detailed procedures of weaving the SNSN are shown in Fig. 3, where $R$ should be smaller than or approximately equal to the radius of the possible conversation zone, while $L$ denotes the span of the CR base-station. The parameter $L$ cannot exceed the maximum communication distance of the CR base-station and is not fixed, but rather is controllable by the CR base-station. As we mentioned above, possible conversation zone, rather than Notalk zone, is easier to be detected by the CR system. Ideally, we hope that wherever the primary system is, all the CR users in that grid could detect it, which also means that maximum distance between two points in the same grid is smaller than the radius of the possible conversation zone. Hence, we can portray the SNSN, as shown in Fig. 3, in detail as follows:

a. Outermost layer: We first choose the parameters for sensing, namely $R, L$ and $\alpha$, by the following formulae and constraints.

$$
\left\{\begin{array}{l}
R^{2}=2 L^{2}(1-\cos \alpha) \\
d_{1}^{2}=2 R^{2}(1-\cos \alpha)
\end{array}\right.
$$

Subject to:

$$
\left\{\begin{array}{l}
R \leq \operatorname{Max}\left(R_{P C}, R_{N T}\right) \\
\alpha=\frac{2 \pi}{N \cdot n} ; \forall N \in \mathbb{N}^{+}
\end{array}\right.
$$

where $\mathrm{n}$ is the number of antennas.

However, since the resolution of the angular division algorithm is constrained by the antenna spacing or sector width, we can not increase the angular resolution arbitrarily. Thus, for a multi-antenna array we should make a tradeoff between the antennas spacing and the angular ambiguity to choose an optimal $N$.

Here, the chord length $R$ is also the maximum distance between two points in the same grids. After that, choose the chord length $R$ as the diagonal of the trapezium. b. Adjacent layer: After the first step, we will get the parameter $d_{1}$ and $R^{\prime}$. Then, we decide $d_{2}$ as follows.

$$
\left\{\begin{array}{l}
\left(R^{\prime}\right)^{2}=2\left(L-d_{1}\right)^{2} \cdot(1-\cos \alpha) \\
R^{2}=d_{2}^{2}+\left(R^{\prime}\right)^{2}-2 \cos \left(\frac{\pi-\alpha}{2}\right) \cdot d_{2} \cdot R^{\prime}
\end{array}\right.
$$

c. Following layers: Carry on deciding the distance parameters $d_{3}$ using equation(3).

$$
\left\{\begin{array}{l}
\left(R^{\prime \prime}\right)^{2}=2\left(L-d_{1}-d_{2}\right)^{2} \cdot(1-\cos \alpha) \\
R^{2}=d_{3}^{2}+\left(R^{\prime \prime}\right)^{2}-2 \cos \left(\frac{\pi-\alpha}{2}\right) \cdot d_{2} \cdot R^{\prime \prime}
\end{array}\right.
$$

d. Judgment: For the $n^{\text {th }}$ step, we should make a judgment before we continue to calculate other parameters.

$$
\begin{cases}\text { Continue } & \text { if } L-d_{1}-d_{2}-\cdots d_{n-1}>R \\ \text { Stop } & \text { if } L-d_{1}-d_{2}-\cdots d_{n-1} \leq R\end{cases}
$$

e. Final decision: When above steps are stopped for satisfying the end condition, we make an additional judgment to decide which grid the central region is in.

$$
\text { If } L-d_{1}-d_{2}-\cdots d_{n-1}>\frac{R}{2}
$$

Then two neighbouring grids are organized as one grid.

$$
\text { If } L-d_{1}-d_{2}-\cdots d_{n-1} \leq \frac{R}{2}
$$

Then all grids neighbouring the CR Base-station are organized as one grid.

\section{How To Sense}

After the SNSN is constructed, the CR base-station knows the grid location of all the CR users. The CR base-station regularly sends sensing commands to all $\mathrm{CR}$ users. Upon receiving a sensing command, the $\mathrm{CR}$ users in the same grid begin to cooperatively detect the presence of a primary system. Then, by Amplify and Forward (AaF) [10] scheme, which amplifies both the signals and the noise and forwards them, they redirect their sensing decisions or information to the CR base-station, in which Equal Gain Combining (EGC) is employed to combine the sensing information for each grid in turn. After that, final decisions about the different grids' occupancy situations are sent to inform all the CR users. The neighboring grids of occupied grids are set as protection guard bands to avoid possible interference from the CR system to the primary system.

The sensing radius of the CR users was not considered when designing the SNSN. One of the major aims of SNSN is to decrease the requirement of detection sensitivity for CR system, which also means the detection radius of the CR users. Ideally, we hope that, after using the SNSN, the requirements for the CR system and the primary system are the same. Thus, our potential assumption is that CR users have, approximately, the same sensing radius as the radius of the possible conversation zone. However, the problem is that, under this assumption, CR users could detect a primary system that is not located in their grid. Our explanation is 
that, even though they are not in the same grid, they could be neighbours. If that happens, for the consideration of reliable detection, setting an additional guard band around these grids should be a good choice.

\section{DIVERSITY RECEPTION IN THE SNSN}

As mentioned in section II, we can make use of the CR basestation to gather sensing information from all the nodes in CR networks. Indeed, in our SNSN CR system, diversity reception can be used to further improve the performance of detection. Diversity combining in our SNSN system means that two or more branches' information-bearing signals are combined in the CR base-station in order to increase the overall SNR and finally improve the performance of detection.

Since the AaF scheme has many virtues, e.g. boosts gain and improves the channel capacity for the primary user system, it is used to redirect both boosted signals and boosted noise to the $\mathrm{CR}$ base-station. The detection of the primary users is a test of binary hypotheses in additive white Guassian noise (AWGN), where $H_{0}$ denotes that the input $Y(n)$ is noise $W[n]$ alone, whereas $H_{1}$ denotes that the input $Y(n)$ is noise $W[n]$ with the presence of a signal $S[n]$ [11]. If there is noise alone, $Y$ follows the central chi-square distribution with 2TWs degree of freedom, while in the presence of primary user, Y follows the non-central chi-square distribution with $2 \mathrm{TW}$ degrees of freedom and non-central parameter $2 \gamma$ [12].

$$
\begin{aligned}
& Y[n]=W[n] ; \quad H_{0} \\
& Y[n]=W[n]+S[n] ; \quad H_{1} \\
& Y \sim \begin{cases}\chi_{2 M}^{2} ; & H_{0} \\
\chi_{2 M}^{2}(2 \gamma) ; & H_{1}\end{cases}
\end{aligned}
$$

Where $M=T W$ indicates time bandwidth products, $T$ is the observation time interval, $W$ is the bandwidth, and $\gamma=S N R$ denotes the Signal-to-Noise Ratio of $Y$. Thus, the Probability Density Function (PDF) of $Y$ can be written as

$$
f_{Y}(y)= \begin{cases}\frac{1}{2^{M} \cdot \Gamma(M)} \cdot y^{M-1} \cdot e^{-\frac{y}{2}} ; & H_{0} \\ \frac{1}{2} \cdot\left(\frac{y}{2 \gamma}\right)^{\frac{M-1}{2}} \cdot e^{-\frac{2 \gamma+y}{2}} \cdot I_{M-1}(\sqrt{2 \gamma y}) ; & H_{1}\end{cases}
$$

where $\Gamma(\bullet)$ is the gamma function and $I_{V}(\bullet)$ is $V^{\text {th }}$ order modified Bessel function.

The Cumulative Distribution Function (CDF) is known to be represented as:

$$
F_{Y}=1-Q_{M}(\sqrt{2 \gamma}, \sqrt{y})
$$

where $Q_{M}(\bullet)$ is the Generalized Marcum Q-Function and can be written as

$$
Q_{M}(a, b)=\int_{b}^{\infty} x\left(\frac{x}{a}\right)^{M-1} e^{-\frac{x^{2}+a^{2}}{2}} I_{M-1}(a x) d x
$$

Thus, the probability of detecting the primary user can be written as follows

$$
P_{d}=P_{r}\left(Y>\lambda \mid H_{1}\right)=Q_{M}(\sqrt{2 \gamma}, \sqrt{\lambda})
$$

where $\lambda$ is the detection threshold.
If the signal $\mathrm{S}(\mathrm{t})$ is transmitted, then the received complex envelopes of signals on the different diversity branches can be written as

$$
r_{k}(t)=g_{k} S_{k}(t)+n_{k}(t), k=1, \ldots, N
$$

where $g_{k}=\alpha_{k} e^{-j \phi_{k}}$ is the fading gain, while $\alpha_{k}$ is the Rayleigh-distributed amplitude and phase angle $\phi_{k}$ is uniformly distributed, and that the AWGN processes $n_{k}(t)$ are independent from branch to branch. Hence, we can represent the SNR within the $k^{\text {th }}$ branch as follows:

$$
\gamma_{\boldsymbol{k}}=\frac{\int_{0}^{T} S_{k}^{2}(t) d t}{N_{k 01}}, k=1, \ldots, N
$$

where $N_{k 01}$ is a one-sided noise power spectral density (PSD) for the $k^{t h}$ branch.

We assume that the magnitude of the received signals are Rayleigh distributed, which makes the SNR $\gamma$ yield an expotential PDF [13] as

$$
f\left(\gamma_{\boldsymbol{k}}\right)=\frac{1}{\bar{\gamma}} e^{-\frac{\gamma_{k}}{\bar{\gamma}}}, k=1, \ldots, N
$$

where $\bar{\gamma}$ is the average SNR in a specific channel.

EGC [14] is often an attractive method of combining due to the fact that it doesn't require the estimation of fading amplitudes and has a relatively low computional complexity compared to Maximum Ratio Combining (MRC). EGC weights every branches equally before combining and sums them to produce the decision. Therefore, EGC decreases the estimation complexity significantly. It can easily be shown that the aggregate SNR at the output of the combiner is given by

$$
\widetilde{\gamma}_{E G C}=\sum_{k=1}^{N} \widetilde{\gamma}_{k}
$$

where the subscript symbol " " denotes an estimated value.

If all branches are independent and identically distributed Rayleigh Fading channels, the PDF of the aggregate SNR at the output of the combiner can be represented as

$$
f_{E G C}(\gamma)=\frac{\gamma^{N-1}}{(N-1) ! \bar{\gamma}^{N}} e^{-\frac{\gamma}{\bar{\gamma}}} \triangleq f(\gamma, \bar{\gamma}, N)
$$

Thus, we can obtain the probability of detection when using the EGC method as

$$
\begin{aligned}
& \bar{P}_{d E G C}=\bar{P}\left(Y>\lambda \mid H_{1}\right)=\int_{0}^{\infty} Q_{M}(\sqrt{2 \gamma}, \sqrt{\lambda}) f_{E G C}(\gamma) d \gamma ; \\
& =\frac{\left(\frac{N}{\gamma}\right)^{N}}{\Gamma(N) 2^{N-1}}\left[\frac { 2 ^ { N - 1 } ( N - 1 ) ! \overline { \gamma } } { ( \frac { N } { \overline { \gamma } } ) ^ { N } ( N + \overline { \gamma } ) } e ^ { - \frac { \lambda N } { 2 ( N + \overline { \gamma } ) } } \left[\left(1+\frac{N}{\bar{\gamma}}\right)\left(\frac{N}{N+\bar{\gamma}}\right)^{N-1} *\right.\right. \\
& \left.L_{N-1}\left(-\frac{\lambda \bar{\gamma}}{2(N+\bar{\gamma})}\right)+\sum_{i=0}^{N-2}\left(\frac{N}{N+\bar{\gamma}}\right)^{i} L_{i}\left(-\frac{\lambda \bar{\gamma}}{2(N+\bar{\gamma})}\right)\right]+ \\
& \left.\Gamma(N)\left(\frac{2 \bar{\gamma}}{N+\bar{\gamma}}\right)^{N} e^{-\frac{\lambda}{2}} \sum_{i=1}^{M-1} \frac{\lambda^{i}}{2^{i+1} i !} \Phi\left(N ; i+1 ; \frac{\lambda \bar{\gamma}}{2(N+\bar{\gamma})}\right)\right]
\end{aligned}
$$

where $\Phi(a ; r ; z)$ is the Confluent Hypergeometric function, while $L_{n}(x)$ is a Laguerre Polynomial. These two functions can be written as

$$
\Phi(a ; r ; z)=1+\frac{a}{r} \frac{z}{1 !}+\frac{a(a+1)}{r(r+1)} \frac{z^{2}}{2 !}+\cdots
$$



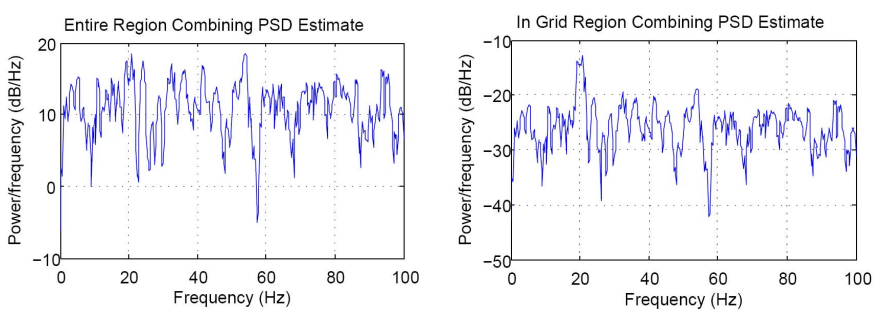

Fig. 4. PSD Estimation
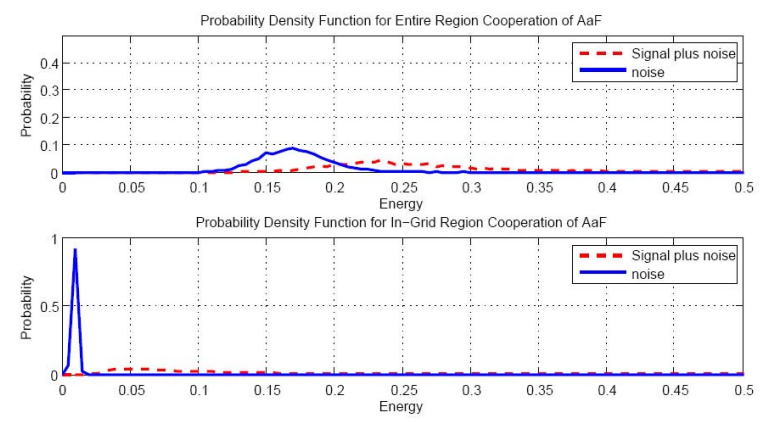

Fig. 5. PDF comparison for No-Grid and In-Grid scheme of AaF

$$
L_{n}(x)=\sum_{m=0}^{n}(-1)^{m}\left(\begin{array}{c}
n \\
n-m
\end{array}\right) \frac{x^{m}}{m !}
$$

The probability of false alarm is given by

$$
\bar{P}_{f E G C}(\lambda) \triangleq \bar{P}_{E G C}\left(Y>\lambda \mid H_{0}\right)=\frac{\Gamma\left(M, \frac{\lambda}{2}\right)}{\Gamma(M)}
$$

\section{NumericAl ANALYSis}

In our experiment, we assume that there are $216 \mathrm{CR}$ nodes uniformly distributed in a circular region, whose radius is about $10 \mathrm{Km}$, and the path-loss exponent is 3.32 . The primary transmitted power is $33 \mathrm{dBm}$ and reference signal power is $25 \mathrm{dBm}$. The primary receiver sensitivity is -10 $\mathrm{dBm}$ and the Carrier frequency is $20 \mathrm{~Hz}$. Firstly, we use the Thompson Multitaper [15] method to estimate the PSD of the entire region combined spectrum and in-grid region combined spectrum. The left hand figure in Fig. 4 shows feature indicating the primary signal is submerged in the noise. However, in the right hand figure, around $20 \mathrm{~Hz}$, it is easier to find the peak of the primary signal, which shows that the SNSN system is in operation. A Monte Carlo method is used to estimate the PDF figures of these two schemes. By introducing the SNSN network, the advantages of in-grid cooperation can be seen in Fig. 5 as it is easier to distinguish between the noise only and the signal plus noise hypotheses.

\section{CONCLUSIONS}

We have modelled the primary system and the CR system by exploring geographical information as a Possible Conversation Zone Model and SNSN, respectively. Furthermore, we have introduced how to use the SNSN to sense the primary system reliably. Additionally, EGC was proposed for combining branches' information in the CR base-station. Simulation results show that the SNSN has a better performance than the no-grid case. Further work will focus on optimizing the detection thresholds for overall combining and local fusion and exploring multi-resolution sensing methods.

\section{ACKNOWLEDGMENT}

The authors would like to thank the supports from the Wolfson Microelectronics Scholarship and the Scottish Funding Council for the Joint Research Institute with HeriotWatt University which is a part of the Edinburgh Research Partnership in Engineering and Mathematics.

\section{REFERENCES}

[1] J. Mitola, "Cognitive radio: an integrated agent architexture for software defined radio", PhD dissertation, Dept. of Teleinformatics, Royal Institute of Technology Stockholm, Sweden. 8 May, 2000.

[2] I. F. Akyildiz, W.-Y.Lee, M. C. Vuran, and S. Mohanty, "Next generation/dynamix spectrum acess/cognitive radio wireless networks: a survey", Computer Networks, vol.50, 2006, pp.2127-2159.

[3] R.W. Brodersen, A. Wolisz, D. Cabric, S.M. Mishra, and D. Willkomm, "Corvsu: a cognitive radio approach for usage of virtual unlicensed spectrum", Berkeley Wireless Research Center (BWRC) White Paper, 2004, pp.1-21.

[4] Simon Haykin, "Cognitive radio: brain-empowered wireless communications", IEEE Journal on Selected Areas in Communications, vol.23, No.2, February, 2005, pp.201-220.

[5] D. Cabric, A. Tkachenko, and R.W. Brodersen, "Spectrum sensing measurements of pilot, energy, and collaborative Detection", in IEEE Military Communications Conference (MILCOM), Oct. 2006.

[6] A. Sahai, N. Hoven, and R. Tandra, "Some fundamental limits on cognitive Radio", in Allerton Conf. Communication, Control, and Computing, Oct. 2004, pp.1-11.

[7] D. Cabric, S. M. Mishra, and R. W. Brodersen, "Implementation issues in spectrum sensing for cognitive radios", in Proc. of Asilomar Conf. on Signals, Systems, and Computers, 2004.

[8] T. Weiss, A. Krohn, F. Capar, I. Martoyo, and F. K. Jondral, "Synchronization algorithms and preamble concepts for spectrum pooling systems", in Proc. of the Mobile and Wireless Communications Summit 2003 (IST) Aveiro, Portugal, June 2003, pp.788-792.

[9] A. Sahai, N. Hoven, S. M. Mishra and R. Tandra, "Fundamental tradeoffs in robust spectrum sensing for opportunistic frequency reuse", Tech Report, also submitted to IEEE JSAC, 2006.

[10] A. Nosratinia, T.E. Hunter, A. Hedayat, "Cooperative communication in wireless networks", IEEE Communications Magazine, vol.42, No.10, Oct. 2004, pp.74- 80 .

[11] M. Gandetto and C. Regazzoni, "Spectrum sensing: a distributed approach for cognitive terminals", IEEE Journal Selected Areas in Communications, vol.25, Apr. 2007, pp.546-557.

[12] H. Urkowitz, "Energy detection of unknown deterministic signals", Proceedings of IEEE, vol.55, Apr. 1967, pp.523-531.

[13] T. Weiss, J. Hillenbrand, A. Krohn, and F. Jondral, "Efficient signaling of spectral resources in spectrum pooling systems", in Proc. of the 10th Symposium on Communications and Vehicular Technology SCVT 2003 Eindhoven, Netherlands, November 2003, pp.1-6.

[14] F. F. Digham, M.-S. Alouini, and M. K. Simon, "On the energy detection of unknown signals over fading channels", in Proc. IEEE International Conference on Communications, vol.5, 2003, pp.3575-3579.

[15] David J. Thomson, "Spectrum estimation and harmonic analysis", Proceedings of the IEEE, 70 (1982), pp. 1055-1096. 